\title{
Excited charmed baryon decays and their implications for fragmentation parameters
}

\author{
John K. Elwood \\ California Institute of Technology, Pasadena, California 91125
}

(Received 9 November 1995)

\begin{abstract}
The production of the excited charmed baryon doublet $\Lambda_{c}^{*}$ via fragmentation is studied. An analysis of the subsequent hadronic decays of the doublet within the framework of heavy hadron chiral perturbation theory produces expressions for both the angular distribution of the decay products and the polarization of the final state heavy baryon in terms of various nonperturbative fragmentation parameters. Future experimental investigation of this system will determine these parameters. In addition, recent experimental results are shown to fix one of the parameters in the heavy hadron chiral Lagrangian. [S0556-2821(96)02409-5]
\end{abstract}

PACS number(s): 13.87.Fh, 12.39.Fe, 12.39. Hg, 13.30.Eg

\section{INTRODUCTION}

The production of a heavy quark at high energy via some hard process is a relatively well understood phenomenon, as we may bring the full apparatus of perturbative QCD to bear on the problem. Less well understood is the subsequent fragmentation of the heavy quark to form heavy mesons and baryons. It is the dynamics of this process that we propose to address in this paper. We imagine that a heavy quark with mass $m_{Q} \gg \Lambda_{\mathrm{QCD}}$ is produced on very short time scales in a hard reaction. It then travels out along the axis of fragmentation and hadronizes on a much longer time scale, at distances of order $1 / \Lambda_{\mathrm{QCD}}$. The fractional change in the heavy quark's velocity is therefore of order $\left(\Lambda_{\mathrm{QCD}} / m_{Q}\right)$, and vanishes at leading order in the heavy quark limit. Likewise, the heavy quark spin couples to the light degrees of freedom via the color magnetic moment operator

$$
\frac{1}{m_{Q}} \bar{h}_{v}^{(Q)} \sigma_{\mu \nu} G^{a \mu \nu} T^{a} h_{v}^{(Q)},
$$

which again vanishes in the heavy limit. We may therefore view the initial fragmentation process as leaving the heavy quark velocity and spin unchanged. Notice that, in this limit, the dynamics are also blind to the mass of the heavy quark, which therefore acts as a static color source in its interactions with the light degrees of freedom.

This simple result may not apply to the ultimate products of the strong fragmentation process, however, as was pointed out by Falk and Peskin [1]. Specifically, the polarization of the final state heavy baryons and mesons may not be determined solely by the heavy quark spin, but may depend in addition on the spin of the light degrees of freedom involved in the fragmentation process. This is the case when the initial fragmentation products decay to lower energy heavy baryons and mesons on a time scale long enough to allow interaction between the heavy quark spin and that of the light degrees of freedom. We will find that this is indeed the case in the $\Lambda_{c}^{*}$ system.

In this situation, one must know something about the spin of the light degrees of freedom in order to proceed further. The parity invariance of the strong interactions, coupled with heavy quark spin symmetry, demands that formation of light degrees of freedom with spin $j$ depends only on the magni- tude of the projection of $j$ onto the axis of fragmentation, and not on its sign. That is, transverse may be preferred to longitudinal, but forward may not be preferred to back. Further, the light system may prefer to invest its angular momentum in orbital channels as opposed to spin channels. These preferences are catalogued by a set of fragmentation parameters: $A$ and $\omega_{1}$, defined in [1], and $B$ and $\widetilde{\omega}_{1}$, defined in the following section.

Let us consider a fragmentation process in which light degrees of freedom of spin $j$ are produced. They then associate with the heavy quark spin $s=\frac{1}{2}$ to form a doublet of total spin $J=j \pm \frac{1}{2}$. Two paths now lie open. The doublet (the two members of which have the same decay rate in the heavy quark limit) may decay rapidly enough that heavy quark spin flip processes have no time to occur. Then the doublet states decay coherently, the heavy quark retains its initial polarization in the final states, and the process begins anew with the decay products. On the other hand, heavy quark spin flip processes may have time to occur, in which case the doublet states decay incoherently, and the heavy quark polarization is altered. The two parameters responsible for determining which regime we are in are the total decay rate out of the doublet, $\Gamma$, and the mass splitting between the doublet states, $\Delta$. The splitting $\Delta$ vanishes in the heavy quark limit, and is of the order of the rate for heavy quark spin flip processes within the doublet. We therefore expect that the situation $\Gamma \gg \Delta$ produces overlapping resonances which decay coherently out of the multiplet, and that the opposite extreme $\Gamma \ll \Delta$ allows for incoherent decays and the influence of the spin of the light degrees of freedom.

\section{THE CHARMED BARYON SYSTEM}

In the charmed baryon system, the ground state is obtained by putting the light diquark in an antisymmetric $I=S=0$ state with spin-parity $j^{P}=0^{+}$. This yields the $J^{P}$ $=\frac{1}{2}^{+}$baryon $\Lambda_{c}^{+}$, with mass $2285 \mathrm{MeV}$. Alternatively, the light quarks may form a symmetric $I=S=1$ state with spinparity $j^{P}=1^{+}$. The light spin then couples to that of the heavy quark to produce the symmetric $J^{P}=\left(\frac{3}{2}^{+}, \frac{1}{2}^{+}\right)$doublet $\left(\Sigma_{c}^{*(0,+,++)}, \Sigma_{c}^{(0,+,++)}\right)$ with mass $(2530 \mathrm{MeV}, 2453 \mathrm{MeV})$. Fragmentation through the $\Sigma_{c}^{(*)}$ system has already been considered in [1]; we concern ourselves here with the $J^{P}$ 
$=\left(\frac{3}{2}^{-}, \frac{1}{2}^{-}\right)$doublet $\left(\Lambda_{c 1}^{*}, \Lambda_{c 1}\right)$ that results when the light diquark is an $I=S=0$ state with a single unit of orbital angular momentum. Allowing the light quarks to have both spin and orbital angular momentum produces a tremendous number of states, none of which have been observed to date. We ignore such states in the analysis that follows.

The fragmentation parameters $A, B, \omega_{1}$, and $\widetilde{\omega}_{1}$, may now be defined. $A$ is taken to be the relative probability of producing any of the nine $I=S=1, j^{P}=1^{+}$diquark states during fragmentation relative to that of producing the $I=S=0$, $j^{P}=0^{+}$ground state. $B$ is similarly the probability for producing any of the three $I=S=0, j^{P}=1^{-}$diquark states relative to ground state production. The parameters $\omega_{1}$ and $\widetilde{\omega}_{1}$, on the other hand, encode the orientation of the light diquark angular momentum. The various helicity states of the spin-parity $1^{+}$and $1^{-}$diquarks are populated with the probabilities

$$
P[1]=P[-1]=\frac{\omega_{1}}{2}, \quad P[0]=1-\omega_{1} \quad \text { for } j^{P}=1^{+},
$$

and

$$
P[1]=P[-1]=\frac{\widetilde{\omega_{1}}}{2}, \quad P[0]=1-\widetilde{\omega}_{1} \text { for } j^{P}=1^{-} .
$$

The analysis of the excited $D$ system in [1] has already indicated that $\omega_{3 / 2}$, the analog of $\omega_{1}$ for the light degrees of freedom in the meson sector, is likely close to zero. One might also anticipate, therefore, that $\omega_{1}$ would be close to zero. We will concentrate on $\widetilde{\omega}_{1}$ most heavily in what follows.

The masses of the $\Lambda_{c 1}^{*}$ and $\Lambda_{c 1}$ are naively expected to be split by $\sim\left(\Lambda_{\mathrm{QCD}}^{2} / m_{c}\right) \simeq 30 \mathrm{MeV}$, in fortuitously close agreement with the recently measured values $M_{\Lambda_{c 1}^{*}}=2625 \mathrm{MeV}$ and $M_{\Lambda_{c 1}}=2593 \mathrm{MeV}$ [2]. Decay of the $\Lambda_{c 1}^{*}$ to $\Lambda_{c 1}$ via pion emission is thus kinematically forbidden, and the corresponding electromagnetic transition is very slow compared with strong decays out of the doublet. Indeed, the dominant decay mode of both $\Lambda_{c 1}^{*}$ and $\Lambda_{c 1}$ is to $\Lambda_{c}$ via pion emission. As both $\left(\Lambda_{c 1}^{*}, \Lambda_{c 1}\right)$ and $\Lambda_{c}$ are $I=0$ states, single pion emission is forbidden by isospin conservation, and the dominant modes are $\Lambda_{c 1}^{*} \rightarrow \Lambda_{c} \pi \pi$ and $\Lambda_{c 1} \rightarrow \Lambda_{c} \pi \pi$. The mass differences $\left(M_{\Lambda_{c 1}^{*}}-M_{\Lambda_{c}}\right)=340 \mathrm{MeV}$ and $\left(M_{\Lambda_{c 1}}-M_{\Lambda_{c}}\right)=308$ $\mathrm{MeV}$ are very close to threshold, and the pions produced will be soft. We therefore expect the decays to be accurately described by heavy hadron chiral perturbation theory.

The CLEO Collaboration recently measured the $\Lambda_{c 1}$ width to be $\Gamma_{\Lambda_{c 1}}=3.9_{-1.2-1.0}^{+1.4+2.0} \mathrm{MeV}$, and placed a new upper bound on the $\Lambda_{c 1}^{*}$ width: $\Gamma_{\Lambda_{c 1}^{*}}<1.9 \mathrm{MeV}$ [2]. It is an interesting breakdown of the naive heavy quark approximation that these rates are significantly different. The explanation is that, at leading order in the heavy hadron chiral Lagrangian, $\Lambda_{c 1}^{*}$ is connected to $\Lambda_{c}$ only via an intermediate $\Sigma_{c}^{*}$, whereas $\Lambda_{c 1}$ is connected via an intermediate $\Sigma_{c}$. Kinematics allows the $\Sigma_{c}$, but not the $\Sigma_{c}^{*}$, to go on shell. The $\Lambda_{c 1}$ thus enjoys a resonant amplification of its decay rate. We also note that the rates above place us securely in the regime $\Gamma \ll \Delta$, so that we anticipate interaction of the heavy quark spin with the light degrees of freedom in decays to the $\Lambda_{c}$. This will allow us to shed some light on the parameter $\widetilde{\omega_{1}}$. In the following section, we provide a brief review of heavy hadron chiral perturbation theory before tackling the $\left(\Lambda_{c 1}^{*}, \Lambda_{c 1}\right)$ decays.

\section{HEAVY HADRON CHIRAL PERTURBATION THEORY}

Heavy hadron chiral perturbation theory incorporates aspects of both ordinary chiral perturbation theory and the heavy quark effective theory, and describes the low energy interactions between hadrons containing a heavy quark and the light pseudo Goldstone bosons. It has been discussed previously in a number of papers [3].

For definiteness we consider the charmed baryon system. Members of the ground state $J^{P}=\frac{1}{2}^{+}$antitriplet are destroyed by the velocity dependent Dirac fields $\mathscr{T}_{i}(v)$, where

$$
\mathscr{T}_{1}=\Xi_{c}^{0}, \quad \mathscr{T}_{2}=-\Xi_{c}^{+}, \quad \mathscr{T}_{3}=\Lambda_{c}^{+} .
$$

The symmetric $J^{P}=\frac{1}{2}^{+}$states are destroyed by the Dirac fields $S^{i j}(v)$ with components

$$
\begin{gathered}
S^{11}=\Sigma_{c}^{++}, \quad S^{12}=\sqrt{\frac{1}{2}} \Sigma_{c}^{+}, \quad S^{22}=\Sigma_{c}^{0}, \quad S^{13}=\sqrt{\frac{1}{2}} \Xi_{c}^{+^{\prime}}, \\
S^{23}=\sqrt{\frac{1}{2}} \Xi_{c}^{0^{\prime}}, \quad S^{33}=\Omega_{c}^{0},
\end{gathered}
$$

and their symmetric $J^{P}=\frac{3}{2}^{+}$counterparts by the corresponding Rarita-Schwinger fields $S_{\mu}^{* i j}(v)$. Finally, we define Dirac and Rarita-Schwinger fields $R_{i}(v)$ and $R_{\mu i}^{*}(v)$ to annihilate the $J^{P}=\frac{1}{2}^{-}$and $J^{P}=\frac{3}{2}{ }^{-}$excited antitriplet states respectively. In our analysis the components of interest will be $R_{3}=\Lambda_{c 1}$ and $R_{\mu 3}^{*}=\Lambda_{c 1, \mu}^{*}$.

As the heavy quark mass goes to infinity, the $J=\frac{3}{2}$ and $J=\frac{1}{2}$ members of the sextet and excited antitriplet multiplets become degenerate. It is then useful to combine them to form the superfields $\mathscr{B}_{\mu i}$ and $\mathscr{S}_{\mu}^{j j}$, defined by

$$
\begin{aligned}
& \mathscr{R}_{\mu i}=\sqrt{\frac{1}{3}}\left(\gamma_{\mu}+v_{\mu}\right) \gamma^{5} R_{i}+R_{\mu i}^{*}, \\
& \mathscr{S}_{\mu}^{i j}=\sqrt{\frac{1}{3}}\left(\gamma_{\mu}+v_{\mu}\right) \gamma^{5} S^{i j}+S_{\mu}^{* i j} .
\end{aligned}
$$

If we are to discuss decay by $\pi$ emission, we must also incorporate the pseudo-Goldstone boson octet into our Lagrangian. The Goldstone bosons are a product of the spontaneous breakdown of the chiral flavor symmetry $\mathrm{SU}(3)_{L} \times$ $\mathrm{SU}(3)_{R}$ to $\mathrm{SU}(3)_{V}$, its diagonal subgroup. They appear in the octet

$$
\begin{aligned}
M & =\sum_{a} \pi^{a} T^{a} \\
& =\sqrt{\frac{1}{2}}\left(\begin{array}{ccc}
\pi^{0} / \sqrt{2}+\eta / \sqrt{6} & \pi^{+} & K^{+} \\
\pi^{-} & -\pi^{0} / \sqrt{2}+\eta / \sqrt{6} & K^{0} \\
K^{-} & \bar{K}^{0} & -2 \eta / \sqrt{6}
\end{array}\right),
\end{aligned}
$$


and are conveniently incorporated into the Lagrangian via the dimensionless fields $\Sigma \equiv e^{2 i M / f}$ and $\xi \equiv e^{i M / f}$, where $f=f_{\pi}=93 \mathrm{MeV}$, the pion decay constant, at lowest order in chiral perturbation theory.

The goal is to combine these fields to produce a Lorentz invariant, parity even, heavy quark spin symmetric, and light chiral invariant Lagrangian. To this end, we now assemble various transformation properties of the fields. Under parity, $P$, the superfields transform as

$$
\begin{gathered}
P \mathscr{B}_{\mu}(\vec{r}, t) P^{-1}=\gamma_{0} \mathscr{B}^{\mu}(-\vec{r}, t), \\
P \mathscr{S}_{\mu}(\vec{r}, t) P^{-1}=-\gamma_{0} \mathscr{S}^{\mu}(-\vec{r}, t), \\
P \mathscr{T}(\vec{r}, t) P^{-1}=\gamma_{0} \mathscr{T}(-\vec{r}, t) .
\end{gathered}
$$

They also obey the constraints

$$
\begin{gathered}
v^{\mu} \mathscr{R}_{\mu}=v^{\mu} \mathscr{S}_{\mu}=0 ; \quad \boldsymbol{v} \mathscr{R}_{\mu}=\mathscr{R}_{\mu} ; \\
\boldsymbol{w} \mathscr{S}_{\mu}=\mathscr{S}_{\mu} ; \quad \boldsymbol{v} \mathscr{T}=\mathscr{T} .
\end{gathered}
$$

The Rarita-Schwinger components obey the additional constraints

$$
\gamma^{\mu} \mathscr{R}_{\mu i}^{*}=\gamma^{\mu} \mathscr{S}_{\mu}^{* i j}=0 .
$$

We are also interested in how the various fields transform under chiral SU(3). The $\Sigma$ and $\xi$ fields obey

$$
\begin{gathered}
\Sigma \rightarrow L \Sigma R^{\dagger}, \\
\xi \rightarrow L \xi U^{\dagger}(x)=U(x) \xi R^{\dagger},
\end{gathered}
$$

where $L$ and $R$ are global $\mathrm{SU}(3)$ matrices, and $U(x)$ is a local member of $\mathrm{SU}(3)_{V}$. If we further define the vector and axial vector fields

$$
\begin{gathered}
V^{\mu}=\frac{1}{2}\left[\xi^{\dagger} \partial^{\mu} \xi+\xi \partial^{\mu} \xi^{\dagger}\right], \\
A^{\mu}=\frac{i}{2}\left[\xi^{\dagger} \partial^{\mu} \xi-\xi \partial^{\mu} \xi^{\dagger}\right],
\end{gathered}
$$

we find that, under chiral SU(3),

$$
\begin{gathered}
V^{\mu} \rightarrow U V^{\mu} U^{\dagger}+U\left(\partial^{\mu} U^{\dagger}\right), \\
A^{\mu} \rightarrow U A^{\mu} U^{\dagger} .
\end{gathered}
$$

The only constraint imposed on the heavy fields is that they transform according to the appropriate sextet or antitriplet representation under transformations of the $\mathrm{SU}(3)_{V}$ subgroup.

There remains one final symmetry to aid us in constructing our Lagrangian, and that is symmetry under reparametrization of the heavy field velocity. The momentum of a heavy hadron is written $p=M v+k$, where $k$ is termed the residual momentum of the hadron. If we make the following shifts in $v$ and $k$ :

$$
v \rightarrow v+\epsilon / M ; \quad k \rightarrow k-\epsilon,
$$

with $v \cdot \epsilon=0$, then $p \rightarrow p$ and $v^{2} \rightarrow v^{2}+O\left(1 / M^{2}\right)$. Therefore, if we are working only to leading order in the (1/M) expan- sion, we demand that our Lagrangian be invariant under such a transformation. The corresponding shifts induced in the fields are [4]

$$
\begin{gathered}
\delta \mathscr{R}_{\mu}=\frac{k}{2 M} \mathscr{R}_{\mu}-\frac{\epsilon^{\nu} \mathscr{R}_{\nu}}{M} v_{\mu}, \\
\delta \mathscr{S}_{\mu}=\frac{k}{2 M} \mathscr{S}_{\mu}-\frac{\epsilon^{\nu} \mathscr{S}_{\nu}}{M} v_{\mu}, \\
\delta \mathscr{T}=\frac{k}{2 M} \mathscr{T} .
\end{gathered}
$$

Invariance of the Lagrangian under these shifts further restricts the terms that may appear, and leaves us with the following form for the most general Lorentz invariant, parity even, heavy quark spin symmetric, and light chiral invariant Lagrangian:

$$
\begin{aligned}
\mathscr{L}_{v}^{(0)}= & \left\{\overline{\mathscr{R}}_{\mu}^{i}\left(-i v \cdot \mathscr{D}+\Delta M_{\mathscr{B}}\right) \mathscr{R}_{i}^{\mu}\right. \\
& +\overline{\mathscr{S}}_{i j}^{\mu}\left(-i v \cdot \mathscr{D}+\Delta M_{\mathscr{S}}\right) \mathscr{S}_{\mu}^{j j}+\overline{\mathscr{T}}^{i} i v \cdot \mathscr{\mathscr { T }} \mathscr{T}_{i} \\
& +i g_{1} \epsilon_{\mu \nu \sigma \lambda} \overline{\mathscr{S}}_{i k}^{\mu} v^{\nu}\left(A^{\sigma}\right)_{j}^{i}(\mathscr{\mathscr { S }})^{j k} \\
& +i g_{2} \epsilon_{\mu \nu \sigma \lambda} \overline{\mathscr{R}}^{\mu i} v^{\nu}\left(A^{\sigma}\right)_{j}^{i}\left(\mathscr{\mathscr { R }}^{\lambda}\right)_{j} \\
& +h_{1}\left[\epsilon_{i j k} \overline{\mathscr{T}}^{i}\left(A^{\mu}\right)_{i}^{j} \mathscr{S}_{\mu}^{k l}+\epsilon^{i j k} \overline{\mathscr{S}}_{k l}^{\mu}\left(A_{\mu}\right)_{j}^{l} \mathscr{T}_{i}\right] \\
& \left.+h_{2}\left[\epsilon_{i j k} \overline{\mathscr{R}}^{\mu i} v \cdot A_{l}^{j} \mathscr{S}_{\mu}^{k l}+\epsilon^{i j k} \overline{\mathscr{C}}_{k l}^{\mu} v \cdot A_{j}^{l} \mathscr{R}_{\mu i}\right]\right\},
\end{aligned}
$$

where $\Delta M_{\mathscr{B}}=M_{\mathscr{B}}-M_{\mathscr{T}}$ is the mass splitting between the excited and ground state antitriplets, and $\Delta M_{\mathscr{S}}=M_{\mathscr{S}}-M_{\mathscr{T}}$ is the corresponding splitting between the sextet and the ground state antitriplet.

In defining the velocity dependent heavy fields which appear above, a common mass must be scaled out of all heavy fields

$$
H=e^{-i M v \cdot x} H_{v},
$$

despite the different masses of the various heavy baryons. In the above analysis we have chosen $M=M_{\Lambda_{c}}$.

It is also instructive at this point to examine the term proportional to $h_{2}$, which allows single $\pi$ transitions between the excited antitriplet and sextet states. This term induces only $S$-wave transitions, although naive angular momentum and parity arguments would allow $D$-wave transitions as well. The $D$-wave transitions are induced by a higher dimension operator which is therefore suppressed by further powers of $M$ and does not appear at leading order in the heavy hadron Lagrangian. This absence of $D$-wave transitions simplifies the way in which the $\pi$ distributions depend on $\widetilde{\omega}_{1}$ in the $\Lambda_{c 1}^{(*)}$ decay process. Finally, we comment quickly on the errors induced by keeping only leading order terms. The relevant expansion parameter in our analyses is $\left(p_{\pi} / M\right)$, so that we expect our results to be valid to $\sim(200 / 2285) \simeq 10 \%$. 


\section{THE PARAMETER $h_{2}$}

The term proportional to $h_{2}$ in the leading order Lagrangian is responsible for the tree-level decay $\Lambda_{c 1} \rightarrow \Sigma_{c} \pi$, the rate for which is easily calculated to be

$$
\begin{aligned}
\Gamma\left(\Lambda_{c 1} \rightarrow \Sigma_{c} \pi\right)= & \frac{\left|h_{2}\right|^{2}}{4 \pi f^{2}} \frac{M_{\Sigma_{c}}}{M_{\Lambda_{c 1}}}\left(M_{\Lambda_{c 1}}-M_{\Sigma_{c}}\right)^{2} \\
& \times \sqrt{\left(M_{\Lambda_{c 1}}-M_{\Sigma_{c}}\right)^{2}-m_{\pi}^{2}},
\end{aligned}
$$

as was done previously in [4]. The $\Sigma_{c}$ may then decay to $\Lambda_{c} \pi$ through the term proportional to $h_{1}$, producing a decay rate $\Gamma\left(\Lambda_{c 1} \rightarrow \Lambda_{c} \pi \pi\right)$ that scales like the combination $\left|h_{1}\right|^{2}\left|h_{2}\right|^{2}$. A quick calculation allows us to express $\left|h_{1}\right|^{2}$ in terms of the partial width $\Gamma\left(\Sigma_{c} \rightarrow \Lambda_{c} \pi\right)$,

$\Gamma\left(\Sigma_{c} \rightarrow \Lambda_{c} \pi\right)=\frac{\left|h_{1}\right|^{2}}{12 \pi f^{2}} \frac{M_{\Lambda_{c}}}{M_{\Sigma_{c}}}\left[\left(M_{\Sigma_{c}}-M_{\Lambda_{c}}\right)^{2}-m_{\pi}^{2}\right]^{3 / 2}$,

which is by far the dominant contribution to $\Gamma_{\Sigma_{c}}$. We may therefore view $\Gamma\left(\Lambda_{c 1} \rightarrow \Lambda_{c} \pi \pi\right)$ as a function of $h_{2}$ and $\Gamma_{\Sigma_{c}}$. This decay is dominated by the pole region where $\Sigma_{c}$ is close to being on shell, and its rate coincides with that for $\Lambda_{c 1} \rightarrow \Sigma_{c} \pi$ as $\Gamma_{\Sigma_{c}} \rightarrow 0$. In this narrow width approximation, we obtain

$$
\Gamma\left(\Lambda_{c 1} \rightarrow \Lambda_{c} \pi^{+} \pi^{-}\right)=4.6\left|h_{2}\right|^{2} \mathrm{MeV} .
$$

The result is modified slightly if we allow the $\Sigma_{c}$ to have a finite width. The $\Sigma_{c}$ is not expected to have a width greater than a few MeV. Setting $\Gamma_{\Sigma_{c}}=2 \mathrm{MeV}$, we find

$$
\Gamma\left(\Lambda_{c 1} \rightarrow \Lambda_{c} \pi^{+} \pi^{-}\right)=4.2\left|h_{2}\right|^{2} \mathrm{MeV}
$$

Comparison with the CLEO measurement [2]

$$
\Gamma\left(\Lambda_{c 1} \rightarrow \Lambda_{c} \pi^{+} \pi^{-}\right)=3.9_{-1.2-1.0}^{+1.4+2.0} \mathrm{MeV}
$$

then yields a central value of $\left|h_{2}\right| \simeq 0.9$ in the narrow width approximation, or $\left|h_{2}\right| \simeq 1.0$ with $\Gamma_{\Sigma_{c}}=2 \mathrm{MeV}$.

\section{PRODUCTION AND DECAY OF $\Lambda_{c 1}$ AND $\Lambda_{c 1}^{*}$}

The probabilities for fragmentation to the $\Lambda_{c 1}$ and $\Lambda_{c 1}^{*}$ states of various helicities may be expressed in terms of the parameters $\widetilde{\omega}_{1}$ and $B$ once the initial polarization of the heavy quark is given. For simplicity, we assume that the initial charm quark is completely left-hand polarized in the analysis that follows. With this assumption, the relative populations of the $\Lambda_{c 1}^{*}$ and $\Lambda_{c 1}$ states are

$$
\begin{gathered}
P\left[\Lambda_{c 1}^{*}\right]=\frac{B}{1+A+B}\left[\frac{\widetilde{\omega}_{1}}{2}, \frac{2}{3}\left(1-\widetilde{\omega}_{1}\right), \frac{\widetilde{\omega}_{1}}{6}, 0\right], \\
P\left[\Lambda_{c 1}\right]=\frac{B}{1+A+B}\left[\frac{1}{3}\left(1-\widetilde{\omega}_{1}\right), \frac{1}{3} \widetilde{\omega}_{1}\right],
\end{gathered}
$$

where the helicity states for $\Lambda_{c 1}^{*}$ read $-\frac{3}{2},-\frac{1}{2}, \frac{1}{2}, \frac{3}{2}$ from left to right, and those for $\Lambda_{c 1}$ read $-\frac{1}{2}, \frac{1}{2}$.

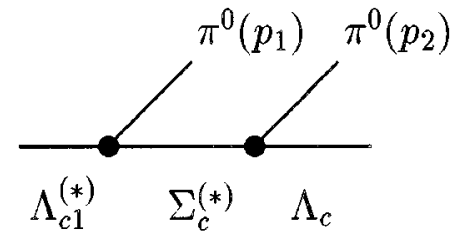

(a)

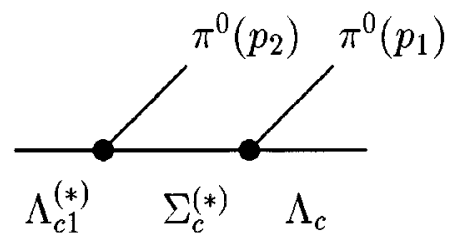

(b)

FIG. 1. Feynman diagrams contributing to $\Lambda_{c 1}^{(*)} \rightarrow \Lambda_{c 1} \pi \pi$ at leading order in the heavy hadron chiral Lagrangian.

We now wish to calculate the double-pion distributions in the decays of these states to the ground state $\Lambda_{c}$. The differential decay rate may be written

$$
\begin{aligned}
\frac{d \Gamma}{d \Omega_{1} d \Omega_{2}}= & \frac{\left|M_{f i}\right|^{2}}{8 M_{\Lambda_{c 1}^{(*)}} M_{\Lambda_{c}}(2 \pi)^{5}} \sqrt{\left(E_{1}^{2}-m_{\pi}^{2}\right)\left(E_{2}^{2}-m_{\pi}^{2}\right)} \\
& \times \delta\left(M_{\left.\Lambda_{c 1}^{(*)}-E_{1}-E_{2}-M_{\Lambda_{c}}\right) d E_{1} d E_{2}}\right.
\end{aligned}
$$

where $\Omega_{1}$ and $\Omega_{2}$ contain the angular variables for the two pions and $E_{1}$ and $E_{2}$ are their energies. A glance at the expression above indicates that we are conserving three momentum, but not energy. The explanation is simply that, in the infinite mass limit, the charm baryon recoils to conserve momentum, but carries off a negligible amount of energy in the process.

Let us first address the case of $\Lambda_{c 1}^{*}$ and $\Lambda_{c 1}$ decay to $\Lambda_{c} \pi^{0} \pi^{0}$. The relevant Feynman diagrams which arise from the Lagrangian (3.21) are shown in Fig. 1. In calculating the decays between $\Lambda_{c 1}^{*}$ and $\Lambda_{c 1}$ states of definite helicity, we find two distinct angular patterns, depending only on the change in the component of spin along the fragmentation axis, $\Delta S_{z}$, between the initial and final state heavy hadrons:

$$
F_{1}\left(\Omega_{1}, \Omega_{2}\right)=\frac{3}{32 \pi^{2}}\left[\cos ^{2} \theta_{1}+\cos ^{2} \theta_{2}+\alpha \cos \theta_{1} \cos \theta_{2}\right]
$$

$$
\begin{aligned}
F_{2}\left(\Omega_{1}, \Omega_{2}\right)= & \frac{3}{64 \pi^{2}}\left[\sin ^{2} \theta_{1}+\sin ^{2} \theta_{2}\right. \\
& \left.+\alpha \sin \theta_{1} \sin \theta_{2} \cos \left(\phi_{2}-\phi_{1}\right)\right]
\end{aligned}
$$

where $\theta_{1}$ and $\theta_{2}$ are the angles between the two pion momenta and the fragmentation axis, and $\phi_{1}$ and $\phi_{2}$ are the azimuthal angles of the pion momenta about this axis. These 


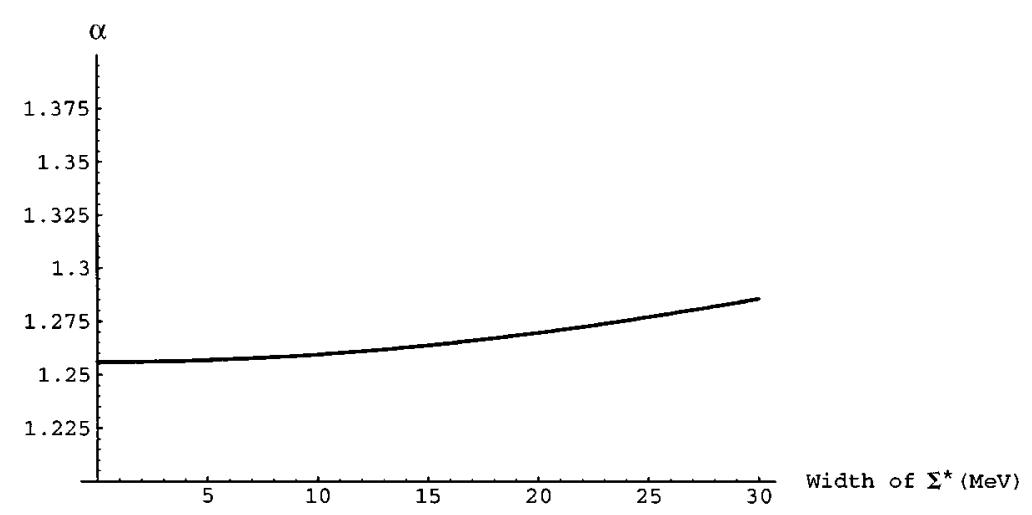

FIG. 2. The variation of the coefficient $\alpha$ as a function of the width of $\Sigma_{c}^{*}$.

angles are defined in the rest frame of the decaying $\Lambda_{c 1}^{(*)}$. The number $\alpha$ arises from interference between the two graphs depicted in Fig. 1, and is defined in (5.6) below. Its dependence on the width $\Gamma_{\Sigma_{c}^{*}}$ is plotted in Fig. 2. To the order we are working, $\alpha=1.3$ for any reasonable value of $\Gamma_{\Sigma *}$ :

$$
\begin{aligned}
& \alpha \equiv \alpha_{1} / \alpha_{2} \text {; } \\
& \alpha_{1}=\int_{m_{\pi}}^{M_{\Lambda_{c 1}^{*}}^{*}-M_{\Lambda_{c}}} d E_{1} \int d E_{2} \delta\left(M_{\Lambda_{c 1}^{*}}-M_{\Lambda_{c}}-E_{1}-E_{2}\right) \\
& \times\left(\frac{2 E_{1} E_{2}\left(E_{1}^{2}-m_{\pi}^{2}\right)\left(E_{2}^{2}-m_{\pi}^{2}\right)\left[\left(M_{\Sigma_{c}^{*}}-M_{\Lambda_{c}}-E_{1}\right)\left(M_{\Sigma_{c}^{*}}-M_{\Lambda_{c}}-E_{2}\right)+\left(\Gamma_{\left.\Sigma_{c}^{*} / 2\right)^{2}}\right]\right.}{\left[\left(M_{\Sigma_{c}^{*}}-M_{\Lambda_{c}}-E 1\right)\left(M_{\Sigma_{c}^{*}}-M_{\Lambda_{c}}-E_{2}\right)+\left(\Gamma_{\Sigma_{c}^{*} / 2}\right)^{2}\right]^{2}+\left(\Gamma_{\Sigma_{c}^{*}} / 2\right)^{2}\left(E_{1}-E_{2}\right)^{2}}\right) ; \\
& \alpha_{2}=\int_{m_{\pi}}^{M_{\Lambda_{c 1}^{*}}-M_{\Lambda_{c}}} d E_{1} \int d E_{2} \delta\left(M_{\Lambda_{c 1}^{*}}-M_{\Lambda_{c}}-E_{1}-E_{2}\right)\left(\frac{E_{1}^{2}\left(E_{2}^{2}-m_{\pi}^{2}\right)^{3 / 2}\left(E_{1}^{2}-m_{\pi}^{2}\right)^{1 / 2}}{\left(M_{\Sigma_{c}^{*}}-M_{\Lambda_{c}}-E_{2}\right)^{2}+\left(\Gamma_{\left.\Sigma_{c}^{*} / 2\right)^{2}}\right.}\right) .
\end{aligned}
$$

The normalized differential rates $(1 / \Gamma)\left(d \Gamma / d \Omega_{1} d \Omega_{2}\right)$ for the various decays are then given in terms of $F_{1}$ and $F_{2}$ by

$$
\begin{gathered}
\frac{1}{\Gamma} \frac{d \Gamma}{d \Omega_{1} d \Omega_{2}}\left\{\left[\Lambda_{c 1}^{*}\left(+\frac{1}{2}\right) \rightarrow \Lambda_{c}\left(+\frac{1}{2}\right)\right],\left[\Lambda_{c 1}^{*}\left(-\frac{1}{2}\right) \rightarrow \Lambda_{c}\left(-\frac{1}{2}\right)\right]\right\}=F_{1}\left(\Omega_{1}, \Omega_{2}\right), \\
\frac{1}{\Gamma} \frac{d \Gamma}{d \Omega_{1} d \Omega_{2}}\left\{\left[\Lambda_{c 1}^{*}\left(+\frac{3}{2}\right) \rightarrow \Lambda_{c}\left(+\frac{1}{2}\right)\right],\left[\Lambda_{c 1}^{*}\left(+\frac{1}{2}\right) \rightarrow \Lambda_{c}\left(-\frac{1}{2}\right)\right],\left[\Lambda_{c 1}^{*}\left(-\frac{1}{2}\right)\right.\right. \\
\left.\left.\rightarrow \Lambda_{c}\left(+\frac{1}{2}\right)\right],\left[\Lambda_{c 1}^{*}\left(-\frac{3}{2}\right) \rightarrow \Lambda_{c}\left(-\frac{1}{2}\right)\right]\right\}=F_{2}\left(\Omega_{1}, \Omega_{2}\right) .
\end{gathered}
$$

The decays $\Lambda_{c 1}^{*}\left( \pm \frac{3}{2}\right) \rightarrow \Lambda_{c}\left(\mp \frac{1}{2}\right)$ are forbidden. A similar calculation for $\Lambda_{c 1}$ decays yields

$$
\begin{aligned}
& \frac{1}{\Gamma} \frac{d \Gamma}{d \Omega_{1} d \Omega_{2}}\left\{\left[\Lambda_{c 1}\left(+\frac{1}{2}\right) \rightarrow \Lambda_{c}\left(+\frac{1}{2}\right)\right],\left[\Lambda_{c 1}\left(-\frac{1}{2}\right) \rightarrow \Lambda_{c}\left(-\frac{1}{2}\right)\right]\right\}=G_{1}\left(\Omega_{1}, \Omega_{2}\right), \\
& \frac{1}{\Gamma} \frac{d \Gamma}{d \Omega_{1} d \Omega_{2}}\left\{\left[\Lambda_{c 1}\left(+\frac{1}{2}\right) \rightarrow \Lambda_{c}\left(-\frac{1}{2}\right)\right],\left[\Lambda_{c 1}\left(-\frac{1}{2}\right) \rightarrow \Lambda_{c}\left(+\frac{1}{2}\right)\right]\right\}=G_{2}\left(\Omega_{1}, \Omega_{2}\right),
\end{aligned}
$$

where

$$
\begin{gathered}
G_{1}=\frac{3}{32 \pi^{2}}\left[\cos ^{2} \theta_{1}+\cos ^{2} \theta_{2}+\beta \cos \theta_{1} \cos \theta_{2}\right] \\
G_{2}=\frac{3}{64 \pi^{2}}\left[\sin ^{2} \theta_{1}+\sin ^{2} \theta_{2}+\beta \sin \theta_{1} \sin \theta_{2} \cos \left(\phi_{2}-\phi_{1}\right)\right]
\end{gathered}
$$

The ratio $\beta$ is defined analogously to $\alpha$ in (5.6), but with the substitutions $M_{\Lambda_{c 1}^{*}} \rightarrow M_{\Lambda_{c 1}}, M_{\Sigma_{c}^{*}} \rightarrow M_{\Sigma_{c}}$, and $\Gamma_{\Sigma_{c}^{*} \rightarrow} \rightarrow \Gamma_{\Sigma_{c}}$, that is, by removing all stars in (5.6). Its dependence on $\Gamma_{\Sigma_{c}}$ is shown in Fig. 3. That $\beta$ is much smaller than $\alpha$ is easily understood. Both $\alpha$ and $\beta$ arise from the interference between Feynman graphs, but in the case of $\Lambda_{c 1}$ decay, the intermediate $\Sigma_{c}$ may go 


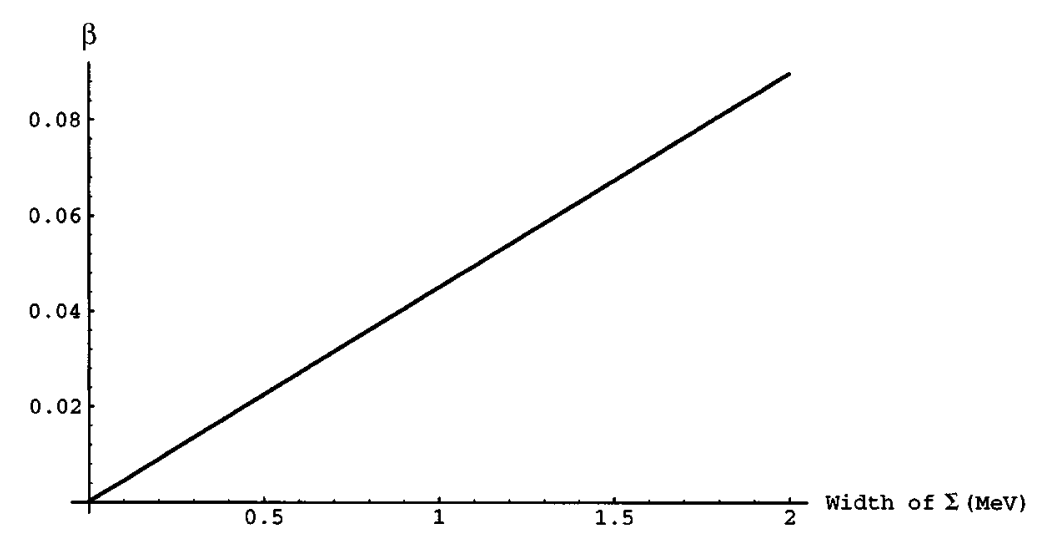

FIG. 3. The variation of the coefficient $\beta$ as a function of the width of $\Sigma_{c}$.

on shell, and in fact, the rate is dominated by this region of phase space. The $\Lambda_{c 1}$ decay is thus essentially a two-step process, and interference effects are therefore relatively unimportant. The steep dependence of $\beta$ on the intermediate state width does not significantly limit our predictions since it is numerically small.

We now take into account the initial populations of the various helicity states, as displayed in (5.1) and (5.2), and allow them to decay incoherently in light of the relation $\Gamma_{\left.\Lambda_{c 1}^{*}\right)} \ll\left(M_{\Lambda_{c 1}^{*}}-M_{\Lambda_{c 1}}\right)$. This produces, after summing final state helicities, the following double pion distributions for decay through $\Lambda_{c 1}^{*}$ and $\Lambda_{c 1}$ states separately:

$$
\begin{aligned}
\frac{1}{\Gamma} \frac{d \Gamma\left(\Lambda_{c 1}^{*} \text { only }\right)}{d \Omega_{1} d \Omega_{2}}= & \frac{3}{32 \pi^{2}}\left\{\left[\frac{1}{3}+\frac{1}{2}\left(\cos ^{2} \theta_{1}+\cos ^{2} \theta_{2}\right)+\frac{2 \alpha}{3} \cos \theta_{1} \cos \theta_{2}+\frac{\alpha}{6} \sqrt{\left(1-\cos ^{2} \theta_{1}\right)\left(1-\cos ^{2} \theta_{2}\right)} \cos \left(\phi_{2}-\phi_{1}\right)\right]\right. \\
& \left.+\widetilde{\omega}_{1}\left[\frac{1}{2}-\frac{3}{4}\left(\cos ^{2} \theta_{1}+\cos ^{2} \theta_{2}\right)-\frac{\alpha}{2} \cos \theta_{1} \cos \theta_{2}+\frac{\alpha}{4} \sqrt{\left(1-\cos ^{2} \theta_{1}\right)\left(1-\cos ^{2} \theta_{2}\right)} \cos \left(\phi_{2}-\phi_{1}\right)\right]\right\}, \\
& \frac{1}{\Gamma} \frac{d \Gamma\left(\Lambda_{c 1} \text { only }\right)}{d \Omega_{1} d \Omega_{2}}=\frac{1}{32 \pi^{2}}\left\{2+\beta\left[\sqrt{\left(1-\cos ^{2} \theta_{1}\right)\left(1-\cos ^{2} \theta_{2}\right)} \cos \left(\phi_{2}-\phi_{1}\right)+\cos \theta_{1} \cos \theta_{2}\right]\right\} .
\end{aligned}
$$

Combining both $\Lambda_{c 1}^{*}$ and $\Lambda_{c 1}$ decays incoherently yields

$$
\begin{aligned}
\frac{1}{\Gamma} \frac{d \Gamma \text { (combined) }}{d \Omega_{1} d \Omega_{2}}= & \frac{1}{32 \pi^{2}}\left\{\left[\frac{4}{3}+\cos ^{2} \theta_{1}+\cos ^{2} \theta_{2}+\left(\frac{4 \alpha}{3}+\frac{\beta}{3}\right) \cos \theta_{1} \cos \theta_{2}+\left(\frac{\alpha}{3}+\frac{\beta}{3}\right) \sqrt{\left(1-\cos ^{2} \theta_{1}\right)\left(1-\cos ^{2} \theta_{2}\right)} \cos \left(\phi_{2}-\phi_{1}\right)\right]\right. \\
& \left.+\widetilde{\omega}_{1}\left[1-\frac{3}{2}\left(\cos ^{2} \theta_{1}+\cos ^{2} \theta_{2}\right)-\alpha \cos \theta_{1} \cos \theta_{2}+\frac{\alpha}{2} \sqrt{\left(1-\cos ^{2} \theta_{1}\right)\left(1-\cos ^{2} \theta_{2}\right)} \cos \left(\phi_{2}-\phi_{1}\right)\right]\right\}
\end{aligned}
$$

Note from Fig. 3 that $\beta$ approaches zero as the width $\Gamma_{\Sigma_{c}}$ vanishes. This means that the double pion distribution (5.14) resulting from $\Lambda_{c 1}$ decay becomes isotropic in this limit. This is easily understood as follows. As $\Gamma_{\Sigma_{c}}$ approaches zero, $\Lambda_{c 1}$ decay is entirely dominated by production of a real intermediate $\Sigma_{c}$ as discussed above, a process which may occur only via $S$-wave pion emission. The subsequent single pion decay of the $\Sigma_{c}$ is also isotropic if $\Lambda_{c}$ helicities are summed over, as previously observed in [1].

Integration of the combined distribution over azimuthal angles produces

$\frac{1}{\Gamma} \frac{d \Gamma \text { (combined) }}{d \cos \theta_{1} d \cos \theta_{2}}=\frac{1}{8}\left\{\left[\frac{4}{3}+\cos ^{2} \theta_{1}+\cos ^{2} \theta_{2}+\left(\frac{4 \alpha}{3}+\frac{\beta}{3}\right) \cos \theta_{1} \cos \theta_{2}\right]+\widetilde{\omega_{1}}\left[1-\frac{3}{2}\left(\cos ^{2} \theta_{1}+\cos ^{2} \theta_{2}\right)-\alpha \cos \theta_{1} \cos \theta_{2}\right]\right\}$,

which is plotted for a variety of $\widetilde{\omega}_{1}$ values in Figs. 4-6.

Alternatively, we may prefer to integrate over pion angles and observe instead the polarization of the final $\Lambda_{c}$. We then find the population ratios

$$
\frac{\Lambda_{c}\left(+\frac{1}{2}\right)}{\Lambda_{c}\left(-\frac{1}{2}\right)}=\frac{2-\widetilde{\omega}_{1}}{4+\widetilde{\omega}_{1}},
$$

for fragmentation through $\Lambda_{c 1}^{*}$ alone,

$$
\frac{\Lambda_{c}\left(+\frac{1}{2}\right)}{\Lambda_{c}\left(-\frac{1}{2}\right)}=\frac{2-\widetilde{\omega}_{1}}{1+\widetilde{\omega}_{1}},
$$




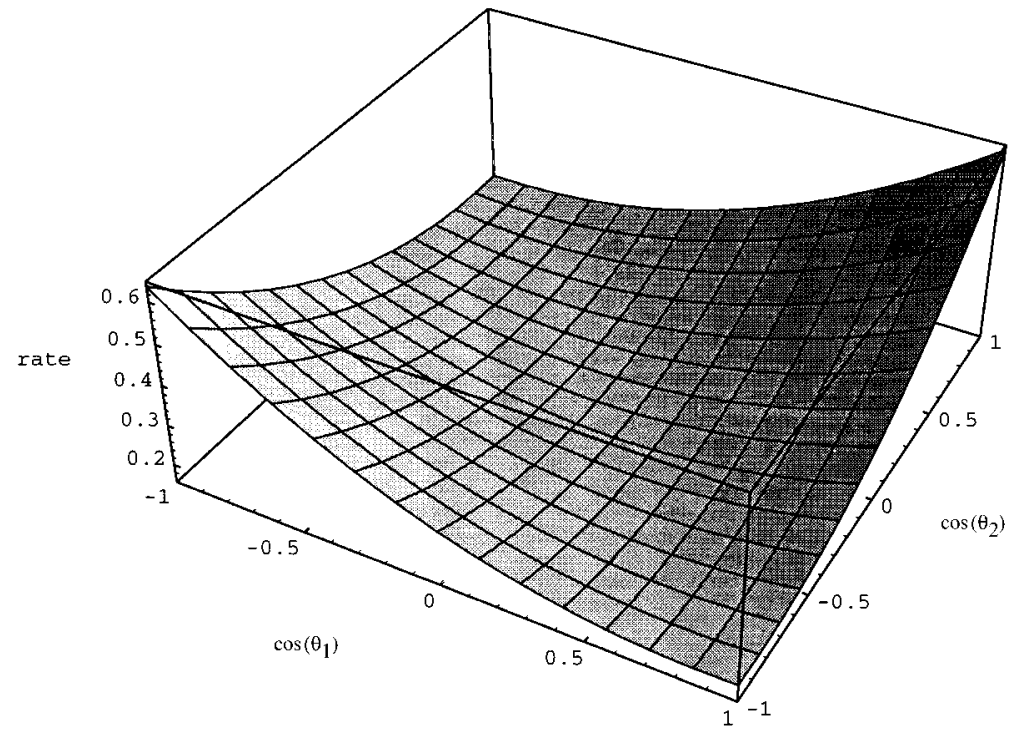

FIG. 4. Normalized differential decay rate for the case $\alpha=1.3, \beta=0.08$, and $\widetilde{\omega}_{1}=0$.

$$
\frac{\Lambda_{c}\left(+\frac{1}{2}\right)}{\Lambda_{c}\left(-\frac{1}{2}\right)}=\frac{4-\widetilde{\omega}_{1}}{5+2 \widetilde{\omega}_{1}}
$$

for the incoherent combination of the two. To be consistent, however, we must include also the effects of initial fragmentation to $\left(\Sigma_{c}^{*}, \Sigma_{c}\right)$ and $\Lambda_{c}$. This analysis was already carried out in [1], and including such effects leaves us with

$$
\frac{\Lambda_{c}\left(+\frac{1}{2}\right)}{\Lambda_{c}\left(-\frac{1}{2}\right)}=\frac{2 A\left(2-\omega_{1}\right)+2 B\left(2-\widetilde{\omega}_{1}\right)}{A\left(5+2 \omega_{1}\right)+B\left(5+2 \widetilde{\omega}_{1}\right)+9} .
$$

We may define the polarization of the final state $\Lambda_{c}$ in terms of the relative production probabilities for $\Lambda_{c}\left(+\frac{1}{2}\right)$ and $\Lambda_{c}\left(-\frac{1}{2}\right)$ as

$$
\mathscr{P}=\frac{\operatorname{Prob}\left[\Lambda_{c}\left(-\frac{1}{2}\right)\right]-\operatorname{Prob}\left[\Lambda_{c}\left(+\frac{1}{2}\right)\right]}{\operatorname{Prob}\left[\Lambda_{c}\left(-\frac{1}{2}\right)\right]+\operatorname{Prob}\left[\Lambda_{c}\left(+\frac{1}{2}\right)\right]} .
$$

For the case of a completely left-handed initial heavy quark, we find

$$
\mathscr{P}=\frac{A\left(1+4 \omega_{1}\right)+B\left(1+4 \widetilde{\omega}_{1}\right)+9}{9(A+B+1)} .
$$

This function may never fall below $\frac{1}{9}$, so that the initial polarization information may never be entirely obliterated by the fragmentation process. As a first guess as to what polarization we may actually expect to measure, we may use the value $\omega_{1}=0$, suggested by experimental study of the charmed meson system [1], and $A=0.45$, the default Lund value $[5,9]$. If we further assume that the light degrees of freedom fragment to $j^{P}=1^{+}$and $j^{P}=1^{-}$states indiscriminately so that $A=B$, we find that $\mathscr{P}$ ranges from 0.58 to 0.79 as $\widetilde{\omega}_{1}$ ranges from 0 to 1 . For a heavy quark with initial polarization $\mathbf{P}$, the above results for $P$ are simply multiplied by $\mathbf{P}$. It is not unreasonable, therefore, to expect a significant fraction of the initial heavy quark's polarization to be observable in the final state $\Lambda_{c}$.

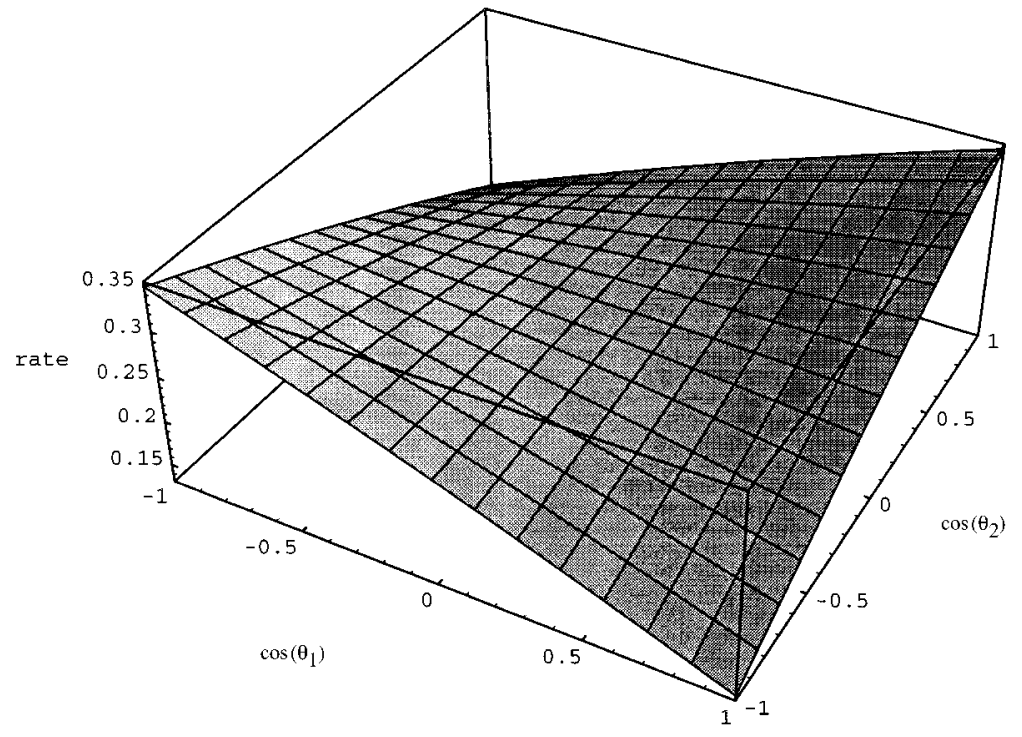

FIG. 5. Normalized differential decay rate for the case $\alpha=1.3, \beta=0.08$, and $\widetilde{\omega}_{1}=0.7$. 


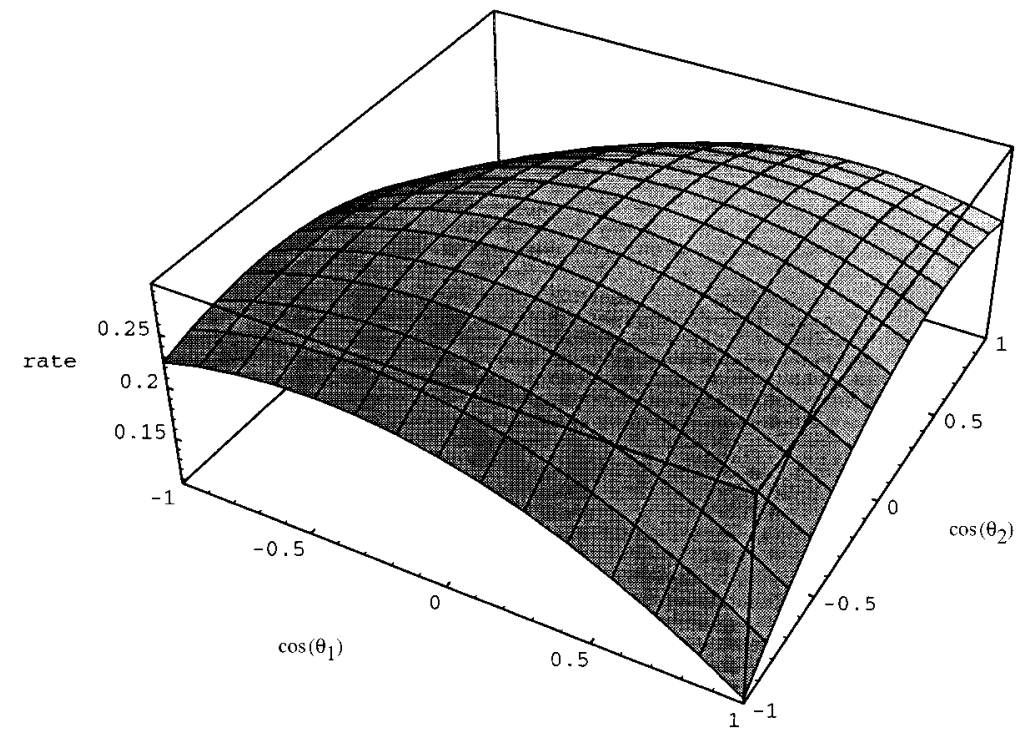

FIG. 6. Normalized differential decay rate for the case $\alpha=1.3, \beta=0.08$, and $\widetilde{\omega}_{1}=1$.
The parameters $A$ and $B$ are also of phenomenological interest. Accurate association of $\Lambda_{c}$ with final state pions should measure the number of zero, one, and two pion events in the ratio:

$$
\Lambda_{c}: \Lambda_{c} \pi: \Lambda_{c} \pi \pi=1: A: B
$$

Information on $A$ and $B$ may also be obtained by measuring the relative number of fragmentation events containing $\Sigma_{c}$ as opposed to those containing $\Sigma_{c}^{*}$. Direct fragmentation to $\left(\Sigma_{c}^{*}, \Sigma_{c}\right)$ produces them in the ratio $\Sigma_{c}^{*}: \Sigma_{c}=2: 1$. This ratio will be diminished, however, by $\Lambda_{c 1}$ that decay to real $\Sigma_{c}$ on their way to $\Lambda_{c}$. The decays of $\Lambda_{c 1}^{*}$ are kinematically forbidden from producing such an enhancement in the $\Sigma_{c}^{*}$ population. In the narrow width approximation for $\Sigma_{c}$, we find

$$
\frac{\text { events with } \Sigma_{c}^{*}}{\text { events with } \Sigma_{c}}=\frac{2}{\left[1+\frac{B}{A}\right]}
$$

An accurate measurement of such departure from naive spin counting could provide information on this interesting ratio, $(B / A)$, and would be especially useful for checking the predictions of various fragmentation models.

A few remarks are in order concerning the decays to $\Lambda_{c} \pi^{+} \pi^{-}$. This case is slightly more complicated than the $\pi^{0} \pi^{0}$ case because the propagator connecting $\Lambda_{c 1}^{*}$ to $\Lambda_{c}$ may be either $\Sigma_{c}^{(*) 0}$ or $\Sigma_{c}^{(*)++}$. This fact, coupled with the different $\Sigma_{c}$ masses,

$$
\begin{aligned}
& M\left[\Sigma_{c}^{++}\right]=2453.1 \pm 0.6 \mathrm{MeV} \\
& M\left[\Sigma_{c}^{+}\right]=2453.8 \pm 0.9 \mathrm{MeV} \\
& M\left[\Sigma_{c}^{0}\right]=2452.4 \pm 0.7 \mathrm{MeV},
\end{aligned}
$$

produces distributions in $\Lambda_{c 1}$ decay that are not symmetric with respect to the $\pi^{+}$and $\pi^{-}$momenta. Indeed, if we boldly accepted the central values of the sigma masses above, we would proceed to calculate an enhancement in the coefficient of $\cos ^{2} \theta_{\pi-}$ by approximately $10 \%$ with respect to that of $\cos ^{2} \theta_{\pi+}$ in (5.4) above, and a similar enhancement for the coefficient of $\sin ^{2} \theta_{\pi-}$ relative to that of $\sin ^{2} \theta_{\pi+}$ in (5.5). In light of the errors listed in (5.25) and the order to which we are working, however, such a conclusion would be inappropriate. The $\pi^{+} \pi^{-}$distributions are, within the accuracy of this calculation, indistinguishable from those of the neutral pions.

\section{CONCLUDING REMARKS}

In this paper, we have studied fragmentation through the $\left(\Lambda_{c 1}^{*}, \Lambda_{c 1}\right)$ system, and have calculated the resultant double pion decay distributions in the well satisfied limit $\Gamma\left(\Lambda_{c 1}^{(*)}\right) \ll\left(M_{\Lambda_{c 1}^{*}}-M_{\Lambda_{c 1}}\right)$. In so doing, we have introduced the fragmentation parameters $\widetilde{\omega}_{1}$ and $B$, and have shown how $\widetilde{\omega}_{1}$ may be extracted from pion angular data. We have also found that the final state $\Lambda_{c}$ particles produced in the fragmentation process should retain a significant fraction of the initial heavy quark's polarization, allowing a test of the standard model's predictions for heavy quark polarization in such hard processes.

Experimental determinations of the $\omega$ parameters are extremely important in testing various ideas about fragmentation. Chen and Wise [6] have estimated $\omega_{3 / 2}$ using the $m_{c} / m_{b} \rightarrow 0$ limit of a perturbative QCD calculation of $b \rightarrow B_{c}^{* *}$ done by Chen [7], and have found that $\omega_{3 / 2}=29 / 114$. That this admittedly oversimplified approach gives reasonable agreement with the experimentally suggested $\omega_{3 / 2}<0.24$ [1] is of significant interest. Yuan [8] has augmented this analysis with a calculation of the dependence of $\omega_{3 / 2}$ on the longitudinal and transverse momentum fractions of the meson. Furthermore, fragmentation models such 
as the Lund model make predictions for parameters related to $A[5,9]$. Similar predictions will be possible for the remaining fragmentation parameters discussed in this paper, in either a limiting case of QCD, or in a model such as Lund, and the experimental extraction of these parameters will therefore provide nontrivial constraints on such methods. Determination of $\widetilde{\omega}_{1}$ may in fact soon be possible at CLEO [10].

\section{ACKNOWLEDGMENTS}

Insightful discussions with Peter Cho, Jon Urheim, Alan Weinstein, and Mark Wise are acknowledged and appreciated. This work was supported in part by the Department of Energy under Contract No. DE-FG03-92-ER40701 (Caltech).
[1] A. F. Falk and M. E. Peskin, Phys. Rev. D 49, 3320 (1993).

[2] CLEO Collaboration, Phys. Rev. Lett. 74, 3331 (1995).

[3] M. Wise, Phys. Rev. D 45, R2188 (1992); G. Burdman and J. Donoghue, Phys. Lett. B 280287 (1992); H. Y. Cheng, C. Y. Cheung, G. L. Lin, Y. C. Lin, T. M. Yan, and H. L. Yu, Phys. Rev. D 46, 1148 (1992); P. Cho, Phys. Lett. B 285145 (1992).

[4] P. Cho, Phys. Rev. D 50, 3295 (1994).
[5] T. Sjöstrand, Comput. Phys. Commun. 39, 347 (1986).

[6] Y. Chen and M. Wise, Phys. Rev. D 50, 4706 (1994).

[7] Y. Chen, Phys. Rev. D 48, 518 (1993).

[8] T. Yuan, Phys. Rev. D 51, 4830 (1995).

[9] B. Andersson, G. Gustafson, G. Ingelman, and T. Sjöstrand, Phys. Rep. 97, 33 (1983).

[10] A. Weinstein (private communication). 